BMJ Surgery, Interventions, \& Health Technologies

\section{A retrospective cohort study on the provision and outcomes of pharmacological therapy after revascularisation for peripheral arterial occlusive disease: a study protocol}

To cite: Peters F, Kreutzburg T, Kuchenbecker J, et al. A retrospective cohort study on the provision and outcomes of pharmacological therapy after revascularisation for peripheral arterial occlusive disease: a study protocol. BMJ Surg Interv Health Technologies 2020;2:e000020. doi:10.1136/ bmjsit-2019-000020

Received 05 August 2019 Revised 22 November 2019 Accepted 10 December 2019

Check for updates

(c) Author(s) (or their employer(s)) 2020. Re-use permitted under CC BY-NC. No commercial re-use. See rights and permissions. Published by BMJ.

${ }^{1}$ Department of Vascular Medicine, University Medical Center Hamburg-Eppendorf, GermanVasc, Hamburg,

Germany

${ }^{2}$ BARMER, Wuppertal, Germany

Correspondence to Dr Christian-Alexander Behrendt;

behrendt@hamburg.de

\section{ABSTRACT}

Background Symptomatic peripheral arterial occlusive disease (PAOD) involves highly complex and costly revascularisations for preventing adverse limb events and impaired survival. Contrary to recommendations from valid guidelines, a large group of patients do not receive adequate pharmacological therapy after such interventions. Based on health insurance claims data, our study aims to assess (1) the extent of provision of pharmacological therapy after revascularisation and (2) related long-term outcomes for all patients and subgroups, that is, gender and disease severity.

Methods A retrospective observational population-based cohort study will be based on data from the second largest statutory health insurance fund in Germany (BARMER) covering about $13 \%$ of the insured population ( 10 million patients). Study entry is the index revascularisation for symptomatic PAOD. Study variables will be analysed and compared among subgroups using parametric and nonparametric tests, generalised linear regression analysis and survival models.

Discussion This study will provide a comprehensive insight in the extent and time trends of adequate provision of pharmacological therapy and long-term outcomes for patients with symptomatic PAOD. This may help to identify those patients benefiting most from improved pharmacological therapy for increasing the success of revascularisations in general.

Trial registration NCT03909022.

\section{BACKGROUND}

Peripheral arterial occlusive disease (PAOD) is a prevalent circulatory condition caused by reduced blood flow in the lower extremities due to atherosclerosis. It affects over 200 million patients worldwide representing a significant burden to healthcare systems with increasing prevalence. ${ }^{12}$ Patients with PAOD are at risk of worsening limb symptoms resulting in major adverse limb events (MALE), major adverse cardiovascular events (MACE) and a generally impaired survival. ${ }^{3}$
For preventing such outcomes, a timely detection and adequate treatment of the disease involving all pillars of invasive and non-invasive vascular care are crucial.

Pharmacological therapy as main pillar of secondary prevention after revascularisation represents an integral element of evidencebased revascularisation. ${ }^{4}$ The rapid progress in the field of endovascular therapy led to an extension of its use in more complex lesions and recently, drug eluting devices have been established in routine care. When compared with the strict regulation of drugs, high risk medical devices in vascular surgery such as drug-eluted or atherectomy devices could be introduced to the market following lower requirements. As a consequence, the lack of randomised and controlled trials led to an increasing utilisation of real-world data to illuminate the benefit, harms and costs of innovative techniques. ${ }^{5}$ Accordingly, the IDEAL recommendations advise the evaluation of the clinical effectiveness of widely adopted interventions by explicitly using routine data focusing on rates and long-term outcomes. ${ }^{5}$ Yet, for any valid comparison of different revascularisation strategies with respect to amputation risk, re-admission and survival, the assessment of post-discharge pharmacological therapy is crucial.

Valid guidelines highlight the importance of pharmacological control of cardiovascular risk factors, in particular of diabetes, hypertension and dyslipidaemia. Additionally, they also recommend the prescription of lipid-lowering and antiplatelet drugs independently from pathological laboratory testing or comorbidities in all patients with PAOD without contraindications (table 1). ${ }^{478}$ 
Table 1 Recent guidelines of the AHA/ACC 2016 and ESC 2017 on the treatment of PAOD

\section{Drugs groups}

Society

Lipid-lowering drugs

Statins are recommended in all patients with PAOD

ESC 2017

In patients with intermittent claudication, on top of general prevention, statins are indicated to improve ESC 2017 walking distance

Treatment with a statin medication is indicated for all patients with PAOD

AHA/ACC 2016

Antithrombotics

Antiplatelet therapy with aspirin alone (range $75-325 \mathrm{mg}$ per day) or clopidogrel alone (75 $\mathrm{mg}$ per day) is recommended to reduce $\mathrm{Ml}$, stroke and vascular death in patients with symptomatic PAOD

In patients with PAOD and AF, OAC is recommended when the CHA2DS2-VASc score is $\geq 2$

AHA/ACC 2016

Cilostazol is an effective therapy to improve symptoms and increase walking distance in patients with

ESC 2017

claudication.

Long-term single-antiplatelet treatment (clopidogrel $75 \mathrm{mg} /$ day or aspirin 75-100 mg/day) is recommended ESC 2017 in patients with symptomatic PAOD

Antiplatelet therapy is recommended in patients with symptomatic PAOD

ESC 2017

Long-term single-antiplatelet treatment (clopidogrel $75 \mathrm{mg} /$ day or aspirin 75-100 mg/day) is recommended ESC 2017 in all patients who have undergone revascularisation

In patients with PAOD who have an indication for OAC (AF, mechanical prosthetic valve), oral anticoagulants alone should be considered

Vitamin $\mathrm{K}$ antagonists may be considered after autologous vein infra-inguinal bypass

ESC 2017

In patients with ALI, systemic anticoagulation with heparin should be administered unless contraindicated.

ESC 2017

Antihypertensives

In patients with PAOD and hypertension, it is recommended to control blood pressure at $<140 / 90 \mathrm{~mm} \mathrm{Hg}$

ESC 2017

Antihypertensive therapy should be administered to patients with hypertension and PAOD to reduce the

AHA/ACC 2016

risk of $\mathrm{Ml}$, stroke, heart failure, and cardiovascular death

The use of ACEi or angiotensin-receptor blockers can be effective to reduce the risk of cardiovascular ischaemic events in patients with PAOD

AHAVACC 2016

ACEi or angiotensin-receptor blockers should be considered as first-line therapy in patients with PAODs and hypertension

ACEi or ARBs should be considered as first-line therapy in patients with PAOD and hypertension

ESC 2017

ESC 2017

ACC, American College of Cardiology; ACEi, ACE inhibitors; AF, atrial fibrillation; AHA, American Heart Association; ALI, acute limb ischaemia; ARBs, angiotensin-receptor blockers; CHA2DS2-VASc, congestive heart failure, hypertension, age, diabetes mellitus, stroke/TIA, vascular disease, age, sex category; ESC, European Society for Cardiology; LEAD, lower extremity artery disease; MI, myocardial infarction; OAC, oral anticoagulants; PAODs, peripheral arterial occlusive disease; SAPT, single-antiplatelet treatment; TIA, transient ischaemic attack.

Depending on the performed type of revascularisation, the patient's risk profile and comorbidities, anticoagulation therapy is also indicated in a narrower patient population. Thereby, uncertainty exists regarding the optimal regime of antithrombotic therapy. ${ }^{3}$ This concerns the indication and optimal duration, particularly the longterm use, of dual-antiplatelet therapy and the combination of single-antiplatelet therapy and anticoagulation (eg, low-dose rivaroxaban and low-dose aspirin).${ }^{910}$ Guidelines emphasise the importance of evidence on comparative effectiveness of pharmacological therapy along the full spectrum of clinical reality, most importantly distinguished by disease severity (intermittent claudication (IC) vs chronic limb threatening ischaemia (CLTI)), obtained in-hospital procedure (endovascular, open surgery, hybrid cases, minor or major amputation) and concomitant comorbidities (eg, atrial fibrillation, coronary artery disease, prior stroke or myocardial infarction). Besides antithrombotic therapy, gaps exist with respect to time trends in prescription rates of lipid-lowering drugs and the optimal dose of such agents in the CLTI subpopulation. ${ }^{4}$

Real-world evidence on the extent of adequate provision of pharmacological therapy and related outcomes is scarce. Prior studies either focused on few medical agents only or they included just a small and selective set of patients with PAOD. Other studies did not distinguish important subgroups, that is, disease severity and gender, or were based on meanwhile outdated data. ${ }^{11-14}$ In general, prior studies reported room for improvement 
in antiplatelet prescription and low statin prescription rates particularly in women. ${ }^{14-16}$

This study aims to determine the provision of pharmacological therapy after revascularisation of PAOD in a real-world setting from a longitudinal and patient-based perspective. First, we focus on extent and time trends in provision of pharmacological therapy. Second, we assess the long-term outcomes MACE, MALE and all-cause mortality. For this purpose, German health insurance claims data will be used. All analyses will be performed for all patients and for subgroups, that is, gender and disease severity.

\section{METHODS}

\section{Study design}

A retrospective cohort study of patients with an index revascularisation for symptomatic PAOD of symptomatic PAOD. The trial was retrospectively registered on clinicaltrials.gov (NCT03909022; Date of registration: April 09, 2019).

\section{Data source}

Analyses will be carried out via remote access to pseudonymised health insurance claims files of BARMER health insurance containing information on about 9.2 million insured persons representing more than 13\% of the insured German population (with about 10000 incident index diagnoses of PAOD annually). ${ }^{1}$ The data extract that we represents the full sample of the BARMER database (calendar years 2005-2018) providing information on all billable services of primary and secondary healthcare in Germany including diagnoses, pharmacological therapy and in-hospital procedures. Diagnoses were coded according to German version of the International Classification of Diseases in its 10th revision and drugs prescriptions according to the German version of the Anatomical Therapeutic Chemical Classification of drugs (table 2). Procedures were identified according to the German Operations and procedure code adapted to the International Classification of Procedures in Medicine.

\section{The German healthcare system}

One of the main priorities of the most recent global vascular guidelines comprises the description of treatment and outcomes of PAOD around the world. ${ }^{4}$ A valid interpretation of the results of our study in this context requires knowledge on the characteristics of the German healthcare system. Generally, it is classified as supplyoriented and choice-oriented public system with a high level of financial and human resources predominantly funded by public financing. ${ }^{17}$ In comparison to other countries, access to resources is not strongly regulated and free choice among providers exist. Provision and remuneration of medical services is subject to fundamentally different rules for outpatient and inpatient care. To date, the prescription of pharmacological therapy is restricted to general practitioners (GPs) and specialists in the ambulatory sector and not linked to a specific indication or primary diagnosis within a fee-for-service scheme. By contrast, inpatient care is based on a diagnosis-relatedgroups payment (DRG), introduced in 2004, with fixed hospital budgets where procedures are mandatory linked to a primary and a set of secondary diagnoses also validated externally. Citizens are universally covered by healthcare with about $90 \%$ statutory health insurance and about $10 \%$ private health insurance. Concerns of overprovision are raised due to a particularly high number of outpatient contacts and number of inpatient stays, especially high admission rate for patients with chronic conditions, and highest per capita expenditures on retail pharmaceuticals. ${ }^{18}$

\section{Table 2 Main variables and coding}

\begin{tabular}{|c|c|c|}
\hline Symptomatic PAOD & Primary inpatient diagnosis, ICD-10 GM, before 2015 & ICD-10 GM, since 2015 \\
\hline $\begin{array}{l}\text { Fontaine II: intermittent } \\
\text { claudication }\end{array}$ & 170.21 & $170.21,170.22$ \\
\hline $\begin{array}{l}\text { Fontaine IV: ulceration } \\
\text { and gangrene }\end{array}$ & $170.23,170.24$ & $170.24,170.25$ \\
\hline Lipid-lowering drugs & $\begin{array}{l}\text { HMG CoA reductase inhibitors, fibrates, bile acid sequestrants, nicotinic } \\
\text { acid and derivatives }\end{array}$ & $\mathrm{C} 10$ \\
\hline Antithrombotics & $\begin{array}{l}\text { VKA, heparins, platelet aggregation inhibitors, enzymes, direct thrombin } \\
\text { inhibitors, direct factor Xa inhibitors }\end{array}$ & B01 \\
\hline $\begin{array}{l}\text { Blood pressure-lowering } \\
\text { drugs }\end{array}$ & $\begin{array}{l}\text { Antihypertensives, diuretics, beta-blocking agents, calcium channel } \\
\text { blockers, agents acting on the renin-angiotensin system }\end{array}$ & $\begin{array}{l}\mathrm{C} 02, \mathrm{C} 03, \mathrm{C} 07, \mathrm{C} 08 \\
\mathrm{C} 09\end{array}$ \\
\hline
\end{tabular}

ATC, anatomical therapeutic chemical classification; HMG CoA, 3-hydroxy-3-methyl-glutaryl-coenzyme A reductase; ICD GM, International classification of diseases German modification; PAOD, peripheral arterial occlusive disease; VKA, Vitamin K antagonists. 


\section{Population}

We will include patients aged $\geq 40$ years with first in-hospital admission for revascularisation of symptomatic PAOD (Fontaine stage II: IC, Fontaine stage III: ischaemic rest pain and Fontaine stage IV: ulceration or gangrene), denoted as index stay (table 2). For finding the index stay and to identify the comorbidities of the patients, up to 5 years of lookback period will be used. After admission from index stay, patients will be followed up until the respective outcome or the end of the observation period. Information on comorbidities were collected from current and past hospital visits, while drug prescriptions will be measured before and after index stay.

\section{Study variables}

Patient characteristics

Patient characteristics will be age at index stay, inpatient diagnosis of PAOD (Fontaine stage II, III and IV), prior outpatient diagnosis and number of drug prescriptions (polypharmacy), Elixhauser comorbidities and van Walraven score, ${ }^{19}$ dyslipidaemia, history of coronary artery disease, myocardial infarction and stroke or transient ischaemic attack.

\section{Characteristics of in-hospital revascularisation}

Variables related to the specific in-hospital revascularisation will be calendar year of index stay, performed invasive procedure (peripheral vascular intervention; PVI, open surgical revascularisation, hybrid procedures using PVI and open-surgical revascularisations, minor and major amputation), length of stay.

\section{Provision of pharmacological therapy}

For determining pharmacological therapy, we will assess the prescription prevalence after index stay grouped in lipid-lowering agents, antithrombotics and antihypertensives (table 2).

\section{Long-term health-related outcomes}

Measured health-related outcomes of interest are allcause mortality, MACE and MALE after index stay.

\section{Analysis}

All analyses will be performed with software SAS V.9.04 (SAS Institute, NC, USA). We will adhere to the reporting of studies conducted using observational routinely collected health data statement and the standardised reporting of secondary data analyses statement for presenting our central findings. ${ }^{20}$ Patient characteristics will be presented in percentage for categorical variables and mean $(+\mathrm{SD})$ or median $(+\mathrm{IQR})$ for continuous variables. Group differences by sex, age, Fontaine stage, invasive procedure will be tested using $\chi^{2}$ test, t-test and Wilcoxon-Mann-Whitney test, relative risk differences, standardised differences and tests for trends over time. For adjusting prescription prevalence, prediction the risk for receiving guideline-specific pharmacological therapy and predicting health-related outcomes generalised linear models will be utilised. As sensitivity analyses, interaction effects will be included in the main analyses and models will be fitted separately to different subgroups. To investigate health-related long-term outcomes and survival, Kaplan-Meier function with log-rank tests and Cox proportional hazard model will be used. We will select covariates to be include in the multivariate models based on clinical relevance and variable selection by forward and backward selection and regularisation approaches. ${ }^{21}$ We will perform complete case deletion for the few patients with missing or implausible information. To adjust for observed and unobserved confounding, propensity score matching and instrumental variables will be applied in models focusing on outcomes.

\section{DISCUSSION}

The particular merit of this study is to provide a comprehensive picture of pharmacological therapy after revascularisation in symptomatic PAOD patients and related outcomes, using a large and diverse cohort. Our analysis will be based on a full sample of health claims data, not suffering the selection bias, recall bias, dropout and panel attrition usually found in other study designs. The findings will complement existing evidence on optimal management of evidence-based revascularisation assisting physicians and patients the choice for particular treatment modalities.

Yet, diagnoses on PAOD, related comorbidities and treatments extracted from claims data were collected for the purpose of reimbursement and not for research. Within the German DRG system, upcoding from cheaper to more expensive diagnoses might be incentivised in the inpatient setting potentially overstating the actual disease severity of the patients to a certain degree. In the outpatient setting, diagnoses are not necessarily linked to treatment because first they were reported once in each quarter only and second, less possibilities for diagnostics exist in general practices. Consequently, some diseases are likely to remain undiagnosed, under-reported or coded less specific. This is undoubtedly relevant for the case of chronic PAOD often associated with none or atypical symptoms during onset and early progression of the disease. However, the longitudinal perspective of our study following each patient for more than a decade, allows to combine information from a longer sequence of multiple visits in the outpatient and inpatient setting providing a much more comprehensive view on individuals' disease progression than a single cross-section alone. Despite the potential bias of upcoding, diagnoses from inpatient care are of high value as they are mandatory, directly linked to a specific procedure at a specific date and cross-checked by a special software and about $20 \%$ reviewed by special physicians. ${ }^{22}$

Patients receive prescriptions for medication either in GP's or specialists' practice and are required to have their prescription filled at registered pharmacies. Data on filled prescriptions are transferred from the pharmacy to the health insurance and approximately $10 \%$ of all 
prescriptions were never filled. ${ }^{23}$ Also, pharmacological therapy dispensed during in-hospital stays and over-thecounter medication is not visible in claims data. Hence, the prescription prevalence as will be reported in our study may slightly underestimate the true prevalence. Additionally, the date of prescription, which will be used in our study, and date of dispense could differ so that the true exposure of pharmacological therapy might be overstated. However, validation studies reported that the date of prescription is more reliable than the date of dispense offsetting the small bias due to the delay in time. ${ }^{23}$ Additionally, validation studies on diagnoses and prescriptions in routine data demonstrated sufficient validity of the data for solid analyses. ${ }^{23}$

A sound construction of patient biographies using data from a single health insurance fund requires to exclude those that entered during the lookback period or left during follow-up aside from the event death. Yet, the potential bias related to this selection problem is likely to be small as the vast majority of people in Germany enters a specific insurance at younger ages and remains there until death. Particularly at older ages, only few people change their insurance company. A related selection problem associated with the use of a single health insurance fund comprises the characteristics of its members compared with the German population: BARMER members are slightly older, the proportion of insured males much lower, better educated and health-related variables slightly below average. ${ }^{24}$ However, compared with other large insurance funds, the data are still more representative for the German population and in any case more representative than RCTs or regional registry or survey studies. As this study focuses only on care practices in Germany, its results need to be understood within the properties of the German healthcare system and are not directly transferable to other countries. For this purpose multi-country analyses should be performed.

While providing rich information on diagnoses, treatment and outcomes, routine data do not contain valid information on more specific clinical parameters and health-related lifestyles as smoking, alcohol consumption, nutrition and obesity and only limited information on socioeconomic status. Thus, a certain amount of variance between relevant subgroups, for example, gender, disease severity and age, will be unexplained pointing at potentials for further studies. Yet, recently developed approaches such as the use of instrumental variables in survival models potentially allow to also adjust for such unobserved confounding, which will be assessed in this study. ${ }^{25}$

Index stay is defined by a first revascularisation for symptomatic PAOD diagnosis using a lookback period of 5 years. Thus, some of the index stays will be artefacts if the patients were diagnosed more than 5 years prior to this date. However, especially chronic and severe diseases were less affected by this issues as demonstrated in validation studies. ${ }^{24}$ To test the validity of this assumption in more detail, we will use shorter and longer lookback times for a subset of patients as sensitivity analysis. Furthermore, we will take outpatient diagnoses into account to assess whether a validated diagnosis could be stated based on the $\mathrm{Q} 1, \mathrm{Q} 2$ or $\mathrm{Q} 3$ criterion $(\mathrm{Q}=$ subsequent quarters where the disease was diagnosed).

Finally, we believe that this study will provide valuable insights in PAOD in a real-world setting, generating hypothesis for further research for improving treatment of the disease.

Twitter Frederik Peters @VASCevidence and Christian-Alexander Behrendt @ VASCevidence

Acknowledgements We are grateful for the scientific advice and technical support of the BARMER.

Contributors The concept of the study was developed by CAB. FP, TK and CAB implemented the study design and statistical analysis plan. FP wrote the first draft of the manuscript and CAB, TK, ESD, JK, UM, HL, and AS reviewed, revised and finalised the manuscript. All authors approved the final version.

Funding This work was supported by the German Joint Federal Committee (grant-no. 01VSF16008 and 01VSF18035) within the IDOMENE0 and RABATT projects. The GermanVasc work group is additionally supported by the German Stifterverband and the CORONA Foundation (grant no. S199/10061/2015), and received research funds from Bayer Vital $\mathrm{GmbH}$, from the foundation of $B$. Braun Melsungen AG, and from the Foundation of the University Heart and Vascular Center Hamburg (UHZ).

Competing interests None declared.

Patient consent for publication Not required.

Ethics approval The initial collection and processing of personal data by BARMER for reimbursement and quality improvement purposes was lawful in accordance to the national regulatory framework and to the European Union General Data Privacy Regulation (EU-GDPR). For further research use, the data were depersonalised by technical matters. Access to the depersonalised data has been granted without the need for consent of the patients based on German Federal Law $§ 67$ social act X.

Provenance and peer review Not commissioned; externally peer reviewed.

Data availability statement There are no data in this work.

Open access This is an open access article distributed in accordance with the Creative Commons Attribution Non Commercial (CC BY-NC 4.0) license, which permits others to distribute, remix, adapt, build upon this work non-commercially, and license their derivative works on different terms, provided the original work is properly cited, appropriate credit is given, any changes made indicated, and the use is non-commercial. See: http://creativecommons.org/licenses/by-nc/4.0/.

ORCID iDs

Frederik Peters http://orcid.org/0000-0001-5832-8410

Christian-Alexander Behrendt http://orcid.org/0000-0003-0406-3319

\section{REFERENCES}

1 Kreutzburg T, Peters F, Rieß HC, et al. Editor's choice - comorbidity patterns among patients with peripheral arterial occlusive disease in Germany: a trend analysis of health insurance claims data. Eur $J$ Vasc Endovasc Surg. In Press 2020;59:59-66.

2 Fowkes FGR, Rudan D, Rudan I, et al. Comparison of global estimates of prevalence and risk factors for peripheral artery disease in 2000 and 2010: a systematic review and analysis. Lancet 2013;382:1329-40.

3 Gerhard-Herman MD, Gornik HL, Barrett C, et al. 2016 AHA/ACC guideline on the management of patients with lower extremity peripheral artery disease: a report of the American College of Cardiology/American heart association Task force on clinical practice guidelines. J Am Coll Cardiol 2017;69:e71-126.

4 Lawrence PF, Gloviczki P. Global vascular guidelines for patients with critical limb-threatening ischemia. J Vasc Surg 2019;69:1S-2.

5 McCulloch P, Altman DG, Campbell WB, et al. No surgical innovation without evaluation: the IDEAL recommendations. Lancet 2009;374:1105-12. 
6 Sedrakyan A, Campbell B, Merino JG, et al. IDEAL-D: a rational framework for evaluating and regulating the use of medical devices. BMJ 2016;353:i2372.

7 Melfi R, Ricottini E. Antiplatelet therapy for peripheral artery disease. Cardiovasc Diagn Ther 2018;8:663-77.

8 Aboyans V, Ricco J-B, Bartelink M-LEL, et al. 2017 ESC guidelines on the diagnosis and treatment of peripheral arterial diseases, in collaboration with the European Society for Vascular Surgery (ESVS): document covering atherosclerotic disease of extracranial carotid and vertebral, mesenteric, renal, upper and lower extremity arteriesEndorsed by: the European Stroke Organization (ESO) The Task Force for the diagnosis and treatment of peripheral arterial diseases of the European Society of Cardiology (ESC) and of the European Society for Vascular Surgery (ESVS). Eur Heart J 2018;39:763-816.

9 Conte MS, Bradbury AW, Kolh P, et al. Global vascular guidelines on the management of chronic limb-threatening ischemia. $J$ Vasc Surg 2019;69:3S-125.

10 Aboyans V, Ricco J-B, Bartelink M-LEL, et al. Editor's Choice - 2017 ESC guidelines on the diagnosis and treatment of peripheral arterial diseases, in collaboration with the European Society for Vascular Surgery (ESVS). Eur J Vasc Endovasc Surg 2018;55:305-68.

11 Dormandy JA, Murray GD. The fate of the claudicant--a prospective study of 1969 claudicants. Eur J Vasc Surg 1991;5:131-3.

12 Goessens BMB, van der Graaf Y, Olijhoek JK, et al. The course of vascular risk factors and the occurrence of vascular events in patients with symptomatic peripheral arterial disease. J Vasc Surg 2007;45:47-54.

13 Pande RL, Perlstein TS, Beckman JA, et al. Secondary prevention and mortality in peripheral artery disease: National health and nutrition examination study, 1999 to 2004. Circulation 2011;124:17-23.

14 McBride CL, Akeroyd JM, Ramsey DJ, et al. Statin prescription rates and their facility-level variation in patients with peripheral artery disease and ischemic cerebrovascular disease: insights from the Department of Veterans Affairs. Vasc Med 2018;23:232-40.
15 Goldstein KM, Zullig LL, Bastian LA, et al. Statin adherence: does gender matter? Curr Atheroscler Rep 2016;18:63.

16 Nastasi DR, Smith JR, Moxon JV, et al. Prescription of pharmacotherapy and the incidence of stroke in patients with symptoms of peripheral artery disease. Stroke 2018;49:2953-60.

17 Reibling N, Ariaans M, Wendt C. Worlds of healthcare: a healthcare system typology of OECD countries. Health Policy 2019;123:611-20.

18 OECD. Germany: country health profile 2017, state of health in the EU. Paris/European Observatory on health systems and policies. Brussels: OECD Publishing, 2017.

19 van Walraven C, Austin PC, Jennings A, et al. A modification of the Elixhauser comorbidity measures into a point system for hospital death using administrative data. Med Care 2009;47:626-33.

20 Swart E, Bitzer E, Gothe H, et al. STandardisierte BerichtsROutine für Sekundärdaten Analysen (STROSA)-ein konsentierter Berichtsstandard für Deutschland, Version 2. Das Gesundheitswesen 2016;78:e145-60.

21 Tibshirani R. Regression shrinkage and selection via the LASSO. J R Stat Soc Series B 1996;58:267-88.

22 Reinecke $\mathrm{H}$, Unrath $\mathrm{M}$, Freisinger $\mathrm{E}$, et al. Peripheral arterial disease and critical limb ischaemia: still poor outcomes and lack of guideline adherence. Eur Heart J 2015;36:932-8.

23 Hoffmann F, Pfannkuche M, Glaeske G. Wie häufig sind Verordnungsund Abgabedatum von Arzneimitteln in Routinedaten korrekt erfasst? Bundesgesundheitsblatt Gesundheitsforschung Gesundheitsschutz 2007;50:1418-23.

24 Hoffmann F, Icks A. [Structural differences between health insurance funds and their impact on health services research: results from the Bertelsmann Health-Care Monitor]. Gesundheitswesen 2012;74:291-7.

25 Dakour-Aridi H, Malas MB. Less biased estimation of the survival benefit of carotid endarterectomy using real-world data: bridging the gap between observational studies and randomized clinical trials. JAMA Netw Open 2018;1:e181831. 